Revista Brasileira de Cartografia

ISSN 1808-0936 | https://doi.org/10.14393/revbrascartogr

Sociedade Brasileira de Cartografia, Geodésia, Fotogrametria e Sensoriamento Remoto

\title{
Avaliação do Impacto da Compatibilização de Referencial das Velocidades na Redução de Coordenadas no PPP
}

\section{Impact of the Compatibility of Velocities Reference Frame in the Process of Updating Coordinates using PPP}

Krisley Xavier Soares de Freitas ${ }^{1}$, William Rodrigo Dal Poz ${ }^{2}$, Lécio Alves Nascimento ${ }^{3}$

1 Universidade Federal de Viçosa (UFV), Departamento de Engenharia Civil, Viçosa - MG, Brasil, krisley.freitas@ufv.br ORCID: https://orcid.org/0000-0001-8453-324X

2 Universidade Federal de Viçosa (UFV), Departamento de Engenharia Civil, Viçosa - MG, Brasil, william.dalpoz@ufv.br ORCID: https://orcid.org/0000-0001-9532-3643

3 Instituto Federal de Educação, Ciência e Tecnologia do Norte de Minas Gerais (IFNMG) - Campus Araçuaí - MG, Brasil, lecio.nascimento@ifnmg.edu.br

ORCID: https://orcid.org/0000-0003-2499-4372

Resumo: No Posicionamento por Ponto Preciso (PPP), as coordenadas estimadas na época do levantamento são referenciadas à materialização do sistema de referência global utilizada nas órbitas dos satélites, atualmente IGb14 (época de coleta dos dados). No entanto, sistemas geodésicos regionais são adotados em diferentes países, como no Brasil, que adota o SIRGAS2000 (época 2000,4). Uma forma de lidar com estas diferenças, é a utilização da transformação de Helmert em conjunto com um modelo de velocidades (que também possui um sistema de referência) para a compatibilização tanto do referencial, quanto da época. Este trabalho tem como objetivo avaliar o desempenho dos modelos VEMOS2009 e VEMOS2017 na aplicação do procedimento de redução de coordenadas para o posicionamento GNSS de alta precisão, considerando ou não a compatibilização do referencial das velocidades obtidas. Utilizou-se dados GNSS em formato RINEX de 175 estações da RBMC processados com o serviço online IBGE-PPP. Diante dos resultados encontrados, pôde-se verificar que a compatibilização de referencial das velocidades se mostrou eficaz para o processo de redução das coordenadas obtidas no IBGE-PPP. Das 1513 coordenadas obtidas com cada uma das quatro velocidades, a VEMOS2009-CCR apresentou 62,92\% das menores discrepâncias, seguida pela VEMOS2017-CCR com 29,54\%, VEMOS2009-SCR com 7,21\% e VEMOS2017-SCR com 0,33\%. Em relação à diminuição das discrepâncias após a compatibilização de referencial das velocidades, verificou-se que, para o VEMOS2009, 85,86\% das coordenadas apresentaram uma menor discrepância após a atualização. Já para o VEMOS2017 essa diminuição ocorreu em 98,61\% das coordenadas.

Palavras-chave: VEMOS2009. VEMOS2017. Redução de coordenadas. Referencial de velocidades. IBGE-PPP.

\begin{abstract}
In Precise Point Positioning (PPP), the coordinates estimated at the epoch of the survey are referenced to the materialization of the global reference system used in the orbits of the satellites, currently IGb14 (epoch of observation). However, regional geodetic systems are officialized and adopted in different countries, such as in Brazil, which adopts SIRGAS2000 (epoch 2000.4). One way of dealing with these differences is to use the Helmert transformation in conjunction with a velocity model (which also has a reference system) to make both the reference and the epoch compatible. This work aims to evaluate the performance of the VEMOS2009 and VEMOS2017 models in the application of the coordinate update procedure for high precision GNSS positioning, considering or not the compatibility of the reference velocities obtained. It was used GNSS data in the RINEX format from 175 RBMC stations processed with the IBGE-PPP online service. In view of the results found, it was possible to verify that the compatibility of the velocities reference proved to be effective for the process of updating the coordinates obtained in the IBGE-PPP. Of the 1513 coordinates obtained with each of the four velocities, VEMOS2009-CCR presented $62.92 \%$ of the smallest discrepancies, followed by VEMOS2017-CCR with 29.54\%, VEMOS2009-SCR with 7.21\% and VEMOS2017-SCR with 0,33\%. Regarding the decrease in discrepancies after the compatibility of the velocities reference, it was found that, for VEMOS2009, 85.86\% of the coordinates presented a smaller discrepancy after the update. For VEMOS2017, this decrease occurred in $98.61 \%$ of the coordinates.
\end{abstract}

Keywords: VEMOS2009. VEMOS2017. Coordinates-update. Reference-frame-of-velocities. IBGE-PPP.

\section{INTRODUÇÃO}


No Brasil, adota-se oficialmente o SIRGAS2000 (Sistema de Referência Geocêntrico para as Américas 2000) como referencial geodésico desde dia 25 de fevereiro de 2005, instituído pela resolução 01/2005 do IBGE (Instituto Brasileiro de Geografia e Estatística). Tal sistema consiste em uma densificação regional do ITRF2000 (International Terrestrial Reference Frame 2000), cuja época de referência é 2000,4 (IBGE, 2015). No entanto, as coordenadas estimadas no PPP (Posicionamento por Ponto Preciso) são originalmente associadas ao referencial das órbitas dos satélites GNSS (Global Navigation Satellite System) para a época do levantamento, atualmente IGb14. Portanto, dados GNSS coletados a partir de 17 de maio de 2020 e processados no serviço online IBGE-PPP terão as coordenadas referenciadas ao IGb14, na época de coleta dos dados (IBGE-PPP, 2020).

Segundo Jekeli (2012), uma forma de lidar com as diferenças de referenciais e épocas das coordenadas consiste em utilizar a transformação de Helmert e seus respectivos parâmetros de transformação, atrelados a um modelo de velocidades para a compatibilização, tanto do sistema de referência (mudança de referencial) quanto da época (redução de coordenadas). Sapucci e Monico (2001) destacam que o rigor com que deve ser tratada as transformações entre os diferentes referenciais no posicionamento de alta precisão é justificável pela ocorrência do movimento das placas tectônicas (deriva continental) que compõem a parte superior da Terra, causando variações de milímetros a centímetros por ano. Consequentemente, uma simples transformação de Helmert não é suficiente, sendo necessário considerar as variações dos parâmetros envolvidos nessa transformação.

Adicionalmente, outros processos geodinâmicos locais ou regionais também exercem influência na movimentação (velocidades) das estações geodésicas, dentre eles: as variações de cargas hidrológicas e atmosféricas (que de modo sazonal, geram alterações de vários centímetros nas coordenadas); terremotos mais intensos, que podem mudar de forma repentina as coordenadas de centímetros a metros etc. (MOREIRA, 2010; SILVA; COSTA; VAZ, 2010; BRUNINI; SÁNCHEZ, 2015).

Desta forma, modelos de velocidades ou de deformações, que englobam alguns dos processos supracitados, podem ser utilizados para determinação das velocidades das estações. Para a região de cobertura do SIRGAS, deve-se utilizar o VEMOS (Modelo de Velocidade para o SIRGAS), em suas versões 2003, 2009, 2015 ou 2017 (SIRGAS, 2020). Como destaca Carvalho (2015), assim como as coordenadas, as velocidades podem estar associadas a diferentes sistemas de referência, sendo a compatibilização de ambas para um referencial comum condição necessária ao procedimento de redução das coordenadas de uma época de origem para uma de destino.

Diversos estudos, afins ou correlatos, que aplicam os procedimentos de redução das coordenadas podem ser encontrados. Dentre estes, pode-se destacar: Soler e Snay (2004) que avaliam a compatibilização dos referenciais ITRF2000 e NAD83 (North American Datum of 1983); Carvalho, Dal Poz e Larocca (2015) que tratam da compatibilização de referenciais de coordenadas e velocidades com estimativa de precisão; Ramos, Dal Poz e Carvalho (2016) onde analisam as possibilidades de compatibilização de referencial e redução de coordenadas estimadas pelo PPP; Braga e Dal Poz (2017) que analisam a compatibilização de referencial e redução de coordenadas entre o ITRF2014 (IGS14) e o SIRGAS2000 e Braga e Dal Poz (2019) que verificam as potencialidades do PPP no Software Bernese com compatibilizações de sistemas geodésicos de referência e de tempo. Com exceção do primeiro, os demais trabalhos utilizam o VEMOS2009 para a determinação das velocidades utilizadas.

Portanto, diante do exposto, objetiva-se neste trabalho avaliar o desempenho dos modelos VEMOS2009 e VEMOS2017 na aplicação do procedimento de redução de coordenadas para o posicionamento GNSS de alta precisão, considerando ou não a compatibilização do referencial das velocidades obtidas.

\section{MODELOS DE VELOCIDADES VEMOS}

O VEMOS consiste em um modelo de velocidades fundamentado nas soluções do sistema de referência SIRGAS, sendo constantemente atualizado. A partir das atualizações foram lançadas algumas versões do VEMOS, denominadas respectivamente de: VEMOS2003 (DREWES; HEIDBACH, 2005), VEMOS2009 (DREWES; HEIDBACH, 2012), VEMOS2015 (SÁNCHEZ; DREWES, 2016) e VEMOS2017 
(DREWES; SÁNCHEZ, 2020). Considerando a recomendação de utilização destes modelos para a área de cobertura do SIRGAS, destaca-se que no Brasil se utiliza, com frequência, o VEMOS2009. Suas vantagens em relação ao VEMOS2003 consistem no maior número de velocidades utilizadas, melhor qualidade das medidas e ampliação da área de abrangência. O VEMOS2017 consiste na atualização mais recente do modelo e descreve a atual deformação na América Latina e no Caribe, dando continuidade ao modelo de superfíciecinemática representado pelo VEMOS2015. Vale destacar que o VEMOS2015 apresenta a limitação de ser válido somente para o período de março de 2010 a abril de 2015 (SÁNCHEZ; DREWES, 2016).

Salienta-se que tais modelos representam médias anuais dos movimentos horizontais da superfície, possibilitando a redução de coordenadas para uma época específica. Devido ao uso neste trabalho, serão descritos apenas os modelos VEMOS2009 e VEMOS2017.

O VEMOS2009 considerou em sua formulação as velocidades de 496 estações, sendo 95 referentes à solução multianual do SIRGAS (SIR09P01) e as demais derivadas de campanhas de rastreio com GPS. A determinação de seu campo de velocidade superficial foi efetuada aplicando-se o método do elemento finito (FEM - Finite Element Method) e colocação por mínimos quadrados (LSC - Least Squares Collocation) associado a funções de covariância, determinadas de forma empírica. Salienta-se que suas velocidades são referenciadas ao ITRF2005 e sua precisão é de aproximadamente 1,5 mm/a (DREWES; HEIDBACH, 2012).

O VEMOS2017 consiste na versão mais atual do modelo, em que descreve a atual deformação na América Latina e no Caribe, recobrindo a região de $120^{\circ} \mathrm{W}, 55^{\circ} \mathrm{S}$ a $35^{\circ} \mathrm{W}, 32^{\circ} \mathrm{N}$, apresentando uma resolução espacial de $1^{\circ} \times 1^{\circ}$. Em sua elaboração utilizou-se as velocidades de 515 estações geodésicas, compreendendo o período 1 de janeiro de 2014 a 28 de janeiro de 2017. Seu campo de velocidade foi determinado com a aplicação do método de colocação por mínimos quadrados associado a funções de covariância, determinadas de forma empírica. Destaca-se que as velocidades determinadas neste modelo estão referenciadas ao ITRF2014 e sua precisão é de 1,0 mm/a na direção norte-sul e 1,7 mm/a na direção leste-oeste (DREWES; SÁNCHEZ, 2020).

IBGE (2015) destaca que os modelos de velocidade supramencionados proporcionam somente a redução das coordenadas planimétricas, uma vez que as variações altimétricas são principalmente decorrentes de movimentos locais, dificultando a modelagem regional.

\section{TRANSFORMAÇÃO DE REFERENCIAL E REDUÇÃO DE COORDENADAS}

A compatibilização entre as materializações dos ITRF (IGS) é efetuada aplicando-se a transformação de Helmert. Os parâmetros utilizados consistem em três translações, três rotações e um fator de escala, assim como suas respectivas taxas de variação, determinados em uma época $t_{k}$, como mostrado na Tabela 1.

Tabela 1 - Parâmetros de transformação entre os ITRF 2014, 2008, 2005 e 2000. *mas: milisegundo de arco

\begin{tabular}{|c|c|c|c|c|c|c|c|}
\hline Parâmetros & $\begin{array}{c}\boldsymbol{T}_{X} \\
(\mathbf{m m})\end{array}$ & $\begin{array}{c}T_{Y} \\
(\mathbf{m m})\end{array}$ & $\begin{array}{c}T_{Z} \\
(\mathbf{m m})\end{array}$ & $\begin{array}{c}D \\
(\mathbf{p p b})\end{array}$ & $\begin{array}{c}\boldsymbol{R}_{X} \\
(\mathbf{m a s})\end{array}$ & $\begin{array}{c}R_{Y} \\
(\mathbf{m a s})\end{array}$ & $\begin{array}{c}R_{Z} \\
(\mathbf{m a s})\end{array}$ \\
\hline ITRF2014 para ITRF2008, época 2010,0 & 1,6 & 1,9 & 2,4 & $-0,02$ & 0,000 & 0,000 & 0,000 \\
\hline ITRF2008 para ITRF2005, época 2000,0 & $-2,0$ & $-0,9$ & $-4,7$ & 0,94 & 0,000 & 0,000 & 0,000 \\
\hline ITRF2005 para ITRF2000, época 2000,0 & 0,1 & $-0,8$ & $-5,8$ & 0,4 & 0,000 & 0,000 & 0,000 \\
\hline Variações temporais (rates) & $\begin{array}{c}\dot{T}_{X} \\
(\mathbf{m m} \\
\text { /ano })\end{array}$ & $\begin{array}{c}\dot{T}_{Y} \\
(\mathbf{m m} \\
\text { /ano) }\end{array}$ & $\begin{array}{c}\boldsymbol{T}_{Z} \\
(\mathbf{m m} \\
\text { /ano) }\end{array}$ & $\begin{array}{c}\dot{D} \\
(\mathbf{p p b} \\
\text { /ano) }\end{array}$ & $\begin{array}{c}\dot{R}_{X} \\
\text { (mas } \\
\text { /ano) }\end{array}$ & $\begin{array}{c}\dot{R}_{Y} \\
\text { (mas } \\
\text { /ano) }\end{array}$ & $\begin{array}{c}\dot{R}_{Z} \\
\text { (mas } \\
\text { /ano) }\end{array}$ \\
\hline ITRF2014 para ITRF2008, época 2010,0 & 0,0 & 0,0 & $-0,1$ & 0,03 & 0,000 & 0,000 & 0,000 \\
\hline ITRF2008 para ITRF2005, época 2000,0 & 0,3 & 0,0 & 0,0 & 0,00 & 0,000 & 0,000 & 0,000 \\
\hline ITRF2005 para ITRF2000, época 2000,0 & $-0,2$ & 0,0 & $-1,8$ & 0,08 & 0,000 & 0,000 & 0,000 \\
\hline
\end{tabular}

Fonte: ALTAMIMI et. al (2007), ALTAMIMI et. al (2012) e ALTAMIMI et. al (2017).

Nesse caso, reduz-se os parâmetros de sua época $\left(t_{k}\right)$ para a época $\left(t_{o}\right)$ referente as coordenadas no sistema de referência de origem (o) conforme Eq. (1) (SAPUCCI; MONICO, 2001; PETTIT; LUZUM, 2010; CARVALHO, 2015): 


$$
\vec{P}_{t_{o}}=\vec{P}_{t_{k}}+\vec{P}_{t_{k}}\left(t_{o}-t_{k}\right) \rightarrow\left(\begin{array}{c}
T_{X} \\
T_{Y} \\
T_{Z} \\
D \\
R_{X} \\
R_{Y} \\
R_{Z}
\end{array}\right)_{t_{o}}=\left(\begin{array}{c}
T_{X} \\
T_{Y} \\
T_{Z} \\
D \\
R_{X} \\
R_{Y} \\
R_{Z}
\end{array}\right)_{t_{k}}+\left(\begin{array}{c}
\dot{T}_{X} \\
\dot{T}_{Y} \\
\dot{T}_{Z} \\
\dot{D} \\
\dot{R}_{X} \\
\dot{R}_{Y} \\
\dot{R}_{Z}
\end{array}\right)_{t_{k}}\left(t_{o}-t_{k}\right)
$$

em que $\vec{P}_{t_{o}}$ é o vetor dos parâmetros de transformação na época das coordenadas no sistema origem; $\vec{P}_{t_{k}}$ é o vetor dos parâmetros de transformação na época de sua determinação; $\vec{P}_{t_{k}}$ é o vetor das taxas de variação dos parâmetros na época de sua determinação; $T_{X}, T_{Y}$ e $T_{Z}$ são as translações em $X, Y$ e $Z$ entre os dois referenciais, dadas em metros; $D$ é o fator de escala entre os dois referenciais, adimensional, dado em partes por bilhão ppb; $R_{X}, R_{Y}$ e $R_{Z}$ são as rotações diferenciais em torno dos eixos $X, Y$ e $Z$, dadas em radianos; e $\dot{T}_{X}, \dot{T}_{Y}, \dot{T}_{Z}, \dot{D}$, $\dot{R}_{X}, \dot{R}_{Y}$ e $\dot{R}_{Z}$ correspondem as taxas de variação anual dos parâmetros anteriormente descritos.

Após a redução dos parâmetros, realiza-se a transformação das coordenadas de um sistema origem $(o)$ para um sistema de destino $(d)$, dada pela Eq. (2), onde as coordenadas nas duas materializações, bem como os sete parâmetros de transformação devem estar na mesma época $t_{o}$ (PETTIT; LUZUM, 2010).

$$
\left[\begin{array}{l}
X \\
Y \\
Z
\end{array}\right]_{d\left(t_{0}\right)}=\left[\begin{array}{l}
X \\
Y \\
Z
\end{array}\right]_{o\left(t_{0}\right)}+\left[\begin{array}{l}
T_{X} \\
T_{Y} \\
T_{Z}
\end{array}\right]_{t_{0}}+\left[\begin{array}{ccc}
D & -R_{Z} & R_{Y} \\
R_{Z} & D & -R_{X} \\
-R_{Y} & R_{X} & D
\end{array}\right]_{t_{0}}\left[\begin{array}{l}
X \\
Y \\
Z
\end{array}\right]_{o\left(t_{0}\right)}
$$

A última etapa do processamento consiste na redução das coordenadas da época $t_{o}$ para a época de interesse $t$. No entanto, para a realização da última etapa, conforme o rigor teórico, faz-se necessário que as velocidades utilizadas estejam no mesmo referencial das coordenadas na época $t_{o}$ (CARVALHO, 2015). Como destacado anteriormente, as velocidades originais do VEMOS2009 são referenciadas ao ITRF2005, e as do VEMOS2017 ao ITRF2014.

A compatibilização do referencial das velocidades do sistema de origem do modelo $\left(o_{m}\right)$ para um sistema destino (d) é dada pela Eq. (3) (SOLER; SNAY, 2004).

$$
\left[\begin{array}{l}
V_{X} \\
V_{Y} \\
V_{Z}
\end{array}\right]_{d}=\left[\begin{array}{l}
V_{X} \\
V_{Y} \\
V_{Z}
\end{array}\right]_{o_{m}}+\left[\begin{array}{l}
\dot{T}_{X} \\
\dot{T}_{Y} \\
\dot{T}_{Z}
\end{array}\right]_{t_{o}}+\left[\begin{array}{ccc}
\dot{D} & -\dot{R}_{Z} & \dot{R}_{Y} \\
\dot{R}_{Z} & \dot{D} & -\dot{R}_{X} \\
-\dot{R}_{Y} & \dot{R}_{X} & \dot{D}
\end{array}\right]_{t_{0}}\left[\begin{array}{l}
X \\
Y \\
Z
\end{array}\right]_{o\left(t_{0}\right)}
$$

Na sequência, reduz-se as coordenadas das estações da época $t_{o}$ para a época $t$ de interesse, utilizandose a Eq. (4) (CARVALHO, 2015; SIRGAS):

$$
\left[\begin{array}{l}
X \\
Y \\
Z
\end{array}\right]_{d(t)}=\left[\begin{array}{l}
X \\
Y \\
Z
\end{array}\right]_{d\left(t_{o}\right)}+\left[\begin{array}{l}
V_{X} \\
V_{Y} \\
V_{Z}
\end{array}\right]_{d}\left(t-t_{o}\right)
$$

em que $[X, Y, Z]_{d(t)}^{T}$ são as coordenadas da estação na época e referencial de interesse; $[X, Y, Z]_{d\left(t_{o}\right)}^{T}$ são as coordenadas cartesianas da estação na época de origem e referencial de interesse; $\left[V_{X}, V_{Y}, V_{Z}\right]_{d}^{T}$ são os vetores velocidade do ponto considerado no mesmo referencial das coordenadas na época $t_{o}$.

\section{METODOLOGIA}

Neste trabalho foram utilizados dados de observação GNSS (GPS e GLONASS) de 175 estações da RBMC, assim como suas coordenadas de referência, referenciadas ao SIRGAS2000, época 2000,4, disponíveis em seus respectivos descritivos. A disposição das estações utilizadas pode ser visualizada na Figura 1. 
Realizou-se três experimentos distintos: o primeiro, utilizando arquivos de observação no formato RINEX, com intervalo de rastreio de 24 horas (L1/L2), das estações ALAR, BOAV, ROGM, SALU, SMAR, TOPL e VICO disponíveis nos dias 01/04 a 10/04 para os anos de 2010 a 2019. Justifica-se a escolha destas estações devido a abrangência de regiões distintas do Brasil; o segundo, utilizando arquivos de observação no formato RINEX, com intervalo de rastreio de 24 horas (L1/L2), de todas as estações disponíveis nos dias 01/04 para todos os anos entre 2010 a 2019; e o terceiro, que além dos dados das sete estações obtidos no primeiro experimento, referentes ao ano de 2019 , utilizou as coordenadas referenciadas ao SIRGAS2000 das mesmas estações na época 2000,4 disponibilizadas diretamente pelo IBGE-PPP.

As observáveis GNSS dos arquivos RINEX foram processadas no serviço online IBGE-PPP (IBGEPPP, 2020), obtendo assim as coordenadas estimadas na data do levantamento. Evidencia-se que em tal serviço, o referencial das coordenadas está associado à data de coleta dos dados, conforme a Tabela 2.

Tabela 2 - Soluções IGS utilizadas no IBGE-PPP, de acordo com a data do levantamento.

\begin{tabular}{cc}
\hline Realização IGS & Data de rastreio GNSS \\
\hline IGS05 & $05 / 11 / 2006$ a $16 / 04 / 2011$ \\
IGS08 & $17 / 04 / 2011$ a 06/10/2012 \\
IGb08 & $07 / 10 / 2012$ a 28/01/2017 \\
IGS14 & $29 / 01 / 2017$ a $16 / 05 / 2020$ \\
IGb14 & $17 / 05 / 2020$ a atualmente \\
\hline
\end{tabular}

Fonte: IBGE (2017) e IBGE-PPP (2020).

Salienta-se que o IGS14/IGb14 e o ITRF2014 são compatíveis e alinhados (IGS ACC, 2020; REBISCHUNG e SCHMID, 2016; REBISCHUNG, 2020), assim como o IGS08/IGb08 e o ITRF2008 (IGS ACC, 2020; WESTON; SOLER, 2012), o IGS05 e o ITRF2005 (IGS ACC, 2020) e o SIRGAS2000 e o ITRF2000 (IGS ACC, 2020; WESTON; SOLER, 2012).

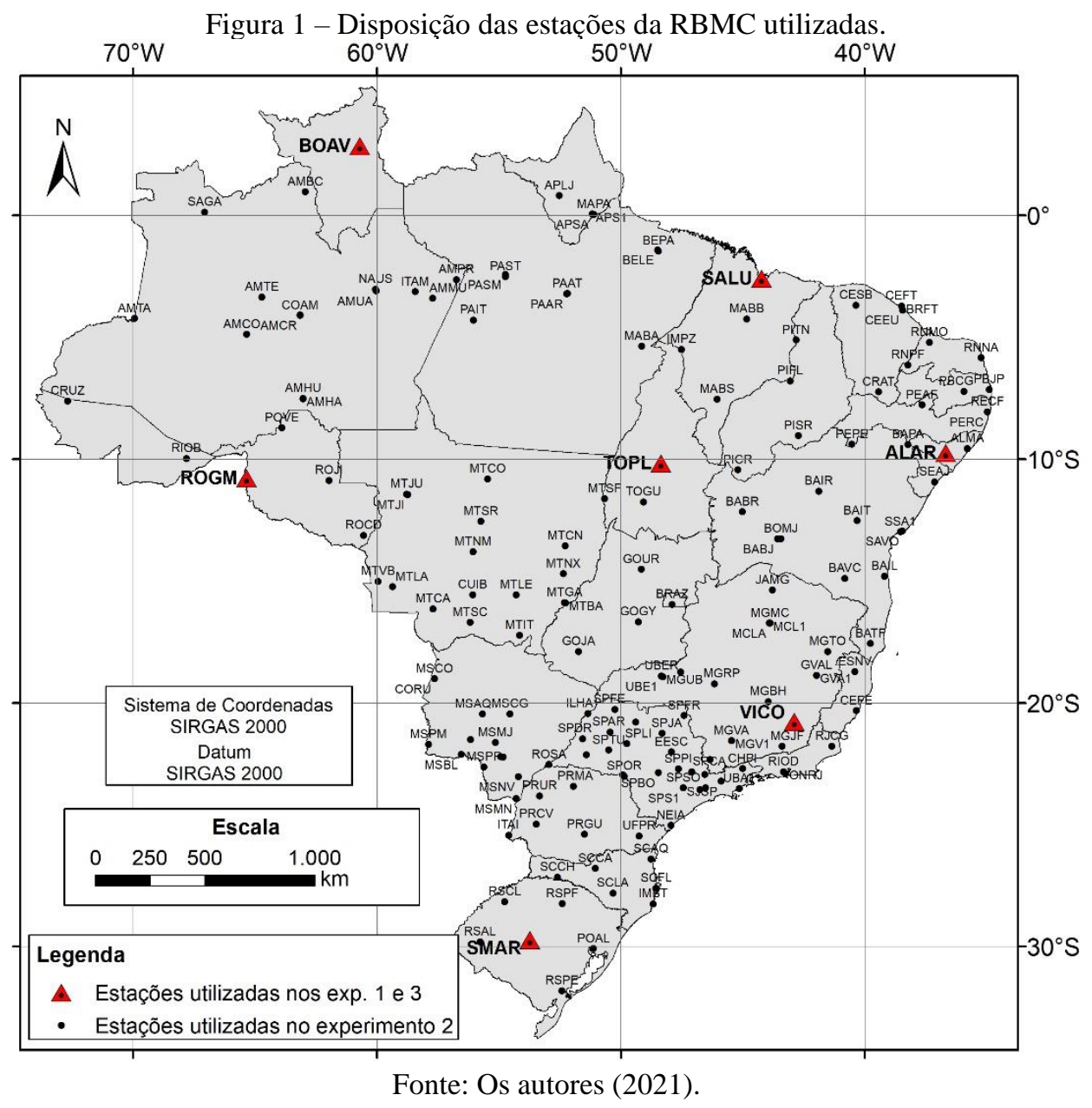

As velocidades de cada estação foram obtidas através da interpolação da grade do VEMOS2009 e 
VEMOS2017, disponibilizados pelo SIRGAS em sua página eletrônica. Este procedimento foi programado em linguagem C, com base na metodologia empregada por Prol et. al (2014).

Após a atualização dos parâmetros e compatibilização dos referenciais das velocidades e das coordenadas, foram obtidas as coordenadas referenciadas ao ITRF2000, época 2000,4, compatíveis com as coordenadas de referência das estações da RBMC.

O procedimento de compatibilização de referenciais se deu seguindo o rigor teórico evidenciado por Carvalho (2015), Almeida, Oliveira e Dal Poz (2016), Braga e Dal Poz (2017) e Ramos, Dal Poz e Carvalho (2016) em que a transformação de referencial ocorre de forma intermediária (transformando do ITRF2014 para ITRF2008, do ITRF2008 para ITRF2005 e finalmente para o ITRF2000) como ilustrado no fluxograma da Figura 2, e não de forma direta (ITRF2014 para ITRF2000). Nesse sentido, após a atualização dos parâmetros e compatibilização dos referenciais das velocidades e das coordenadas, obteve-se as coordenadas referenciadas ao ITRF2000, época 2000,4, compatíveis com as coordenadas de referência das estações da RBMC.

Figura 2 - Fluxograma do procedimento completo para compatibilização de referencial e redução de uma coordenada do ITRF2014, época de coleta dos dados, para o ITRF2000, época 2000,4.

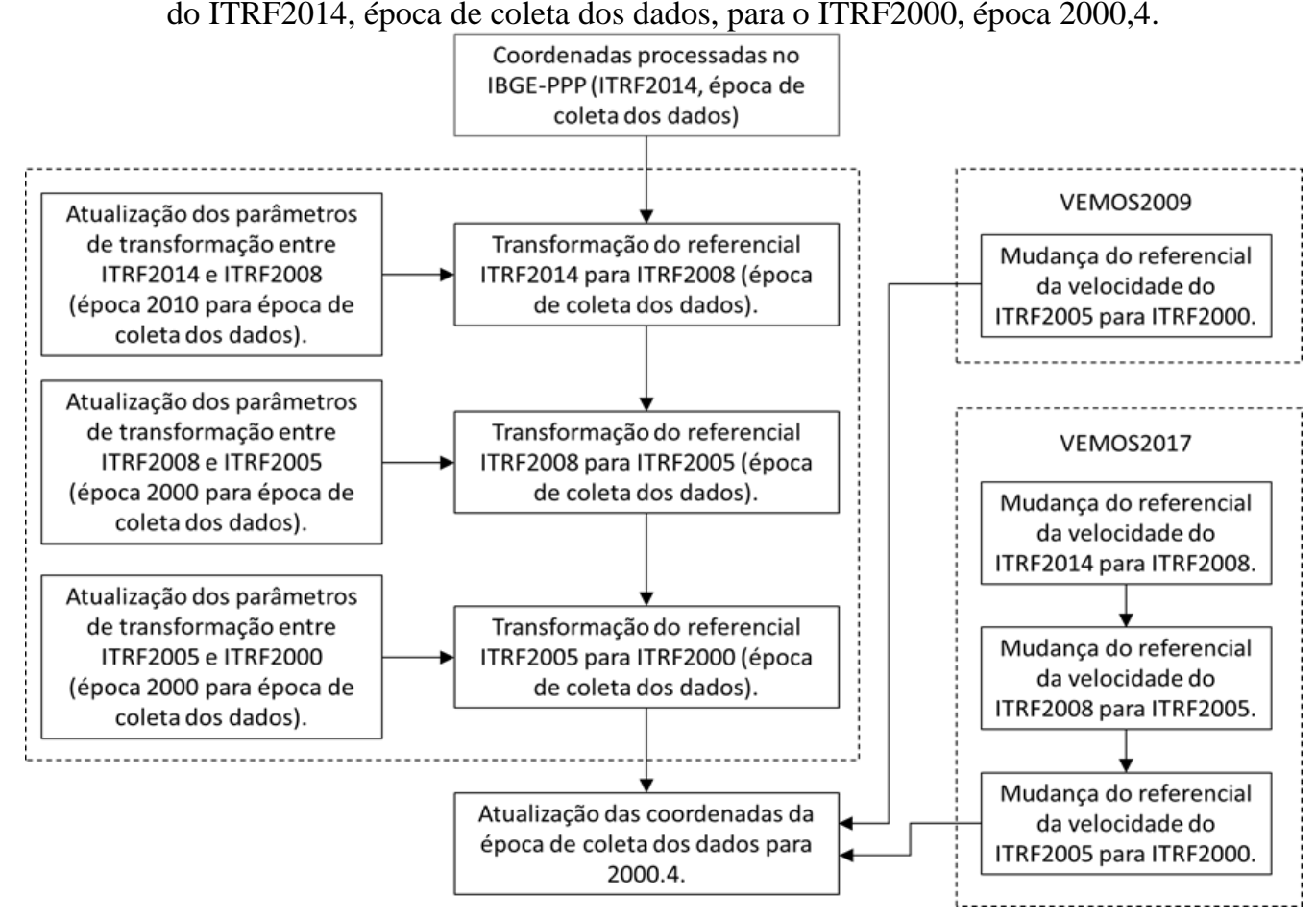

Fonte: Os autores (2021).

Para calcular as discrepâncias planimétricas, primeiro fez-se necessário a transformação das coordenadas cartesianas estimadas em coordenadas geodésicas $(\phi, \lambda, h)$, no mesmo referencial, conforme Monico (2008). Segundo a recomendação de Pettit e Luzum (2010), utilizou-se o elipsoide GRS80 (Geodetic Reference System 1980) na transformação. A sequência de cálculos para a obtenção das discrepâncias planimétricas, em metros, é apresentada nas Eq. (5 a 10) (TORGE, 2001).

Inicialmente, são calculadas as discrepâncias da latitude $(\phi)$ e longitude $(\lambda)$ em radianos (Eq. (5)) e posteriormente em metros (Eq. (6)).

$$
\begin{gathered}
\left|\begin{array}{c}
\Delta \phi(\text { rad }) \\
\Delta \lambda(\text { rad })
\end{array}\right|=\left|\begin{array}{c}
\phi(\text { referência })-\phi(\text { estimada }) \\
\lambda(\text { referência })-\lambda(\text { estimada })
\end{array}\right| \\
\left|\begin{array}{c}
\Delta \phi(\text { metros }) \\
\Delta \lambda(\text { metros })
\end{array}\right|=\left|\begin{array}{c}
M . \Delta \phi(\text { rad }) \\
N . \cos \phi . \Delta \lambda(\text { rad })
\end{array}\right|
\end{gathered}
$$

onde $M$ é o raio de curvatura da seção meridiana (Eq. (7)), $N$ o raio de curvatura da seção primeiro vertical (Eq. (8)), $\bar{\phi}$ é a média entre as latitudes de referência e estimada, e $e^{2}$ a segunda excentricidade (Eq. (9)). 
Destaca-se que as coordenadas de referência foram obtidas nos descritivos das estações da RBMC (SIRGA2000, época 2000,4).

$$
\begin{gathered}
M=\frac{a\left(1-e^{2}\right)}{\left(1-e^{2} \operatorname{sen}^{2} \bar{\phi}\right)^{\frac{3}{2}}} \\
N=\frac{a}{\left(1-e^{2} \operatorname{sen}^{2} \bar{\phi}\right)^{\frac{1}{2}}} \\
e^{2}=\frac{a^{2}-b^{2}}{a^{2}}
\end{gathered}
$$

A discrepância planimétrica, em metros, é dada pela Eq. (10).

$$
T=\sqrt{\Delta \phi_{(\text {metros })}^{2}+\Delta \lambda_{(\text {metros })}^{2}}
$$

Salienta-se que as discrepâncias altimétricas não serão analisadas, uma vez que o processo de atualização de coordenadas é predominantemente horizontal (IBGE, 2015).

\section{RESULTADOS E DICUSSÕES}

As análises foram realizadas sobre as discrepâncias obtidas com o uso dos dois modelos considerando quatro estratégias de processamento: velocidades do VEMOS2009 sem compatibilização de referencial das velocidades (SCR), do VEMOS2009 com compatibilização do referencial das velocidades (CCR), do VEMOS2017-SCR e do VEMOS2017-CCR.

\subsection{Experimento 1}

Os resultados do Experimento 1 referem-se às discrepâncias planimétricas obtidas utilizando as quatro velocidades para cada uma das estações (ALAR, BOAV, ROGM, SALU, SMAR, TOPL e VICO), apresentadas na Figura 3.

Para a estação ALAR pode-se notar que houve uma significativa melhora (diminuição) das discrepâncias considerando o VEMOS2017-CCR. A melhora mais significativa foi de 3,4cm e a menos de 1,0cm, correspondendo a uma redução de $91,91 \%$ e 54,63\% respectivamente, em relação à discrepância obtida no VEMOS2017-SCR. Em média, as discrepâncias apresentaram uma redução de 71,5\% (2,3cm) em relação ao VEMOS2017-SCR. Considerando o VEMOS2009-CCR verificou-se que apenas 48,31\% das discrepâncias melhoraram, com máxima de $1,1 \mathrm{~cm}(66,26 \%)$, mínima de $0,2 \mathrm{~cm}(12,36 \%)$ e média de $0,5 \mathrm{~cm}(30,59 \%)$. Restringindo-se apenas às discrepâncias que apresentaram pior resultado, destaca-se que o mais expressivo foi de $+0,99 \mathrm{~cm}$, o que representou uma piora de $150 \%$, a média foi de $+0,35 \mathrm{~cm}(+35,11 \%)$. Comparando os resultados apresentados pelo VEMOS2017-CCR e VEMOS2009-CCR, pode-se notar que o VEMOS2017CCR apresentou melhores resultados, o que não ocorreu quando comparados o VEMOS2017-SCR e o VEMOS2009-SCR, cujos melhores resultados foram obtidos pelo VEMOS2009-SCR.

A estação BOAV apresentou resultados similares à ALAR para os dois modelos VEMOS, tanto para as velocidades SCR como para as CCR (que melhoraram 100\% das discrepâncias da estação). O VEMOS2017CCR apresentou melhora máxima de 3,3cm (99,34\%), mínima de $1,0 \mathrm{~cm}(45,31 \%)$ e média foi de 2,2cm (80,04\%) quando comparado ao VEMOS2017-SCR. Para o VEMOS2009-CCR, a máxima redução foi de $3,0 \mathrm{~cm}(97,11 \%)$, a menor de $0,5 \mathrm{~cm}(28,14 \%)$ e a média foi de $1,9 \mathrm{~cm}(77,61 \%)$ em relação ao VEMOS2009SCR. Equivalente ao encontrado nas estações BOAV e ALAR, a estação ROGM também apresentou comportamento similar para os cenários considerados. O VEMOS2017-CCR evidenciou redução máxima de 
2,7cm (92,91\%), mínima de $0,1 \mathrm{~cm}(2,37 \%)$ e média de 1,4cm (48,21\%), quando comparado ao VEMOS2017SCR. Para o VEMOS2009-CCR, a melhora máxima foi de 2,6cm (95,56\%), a mínima de $0,1 \mathrm{~cm}(2,74 \%)$ e a média foi de $1,4 \mathrm{~cm}(47,12 \%)$ comparado ao VEMOS2009-SCR.

Figura 3 - Comparação das discrepâncias planimétricas das estações ALAR, BOAV, ROGM, SALU, SMAR, TOPL e VICO obtidas utilizando as quatro velocidades.

VEMOS2017 vs VEMOS2009 - Dados ALAR 01/04 a

10/04 de 2010 a 2019

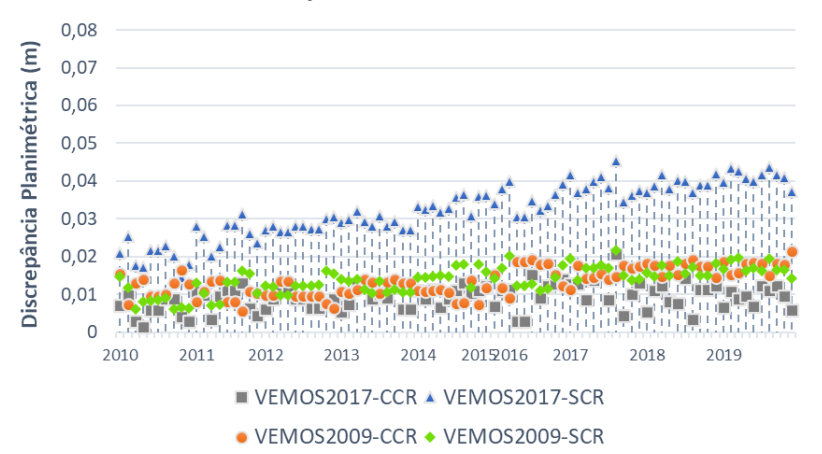

VEMOS2017 vs VEMOS2009 - Dados BOAV 01/04 a 10/04 de 2010 a 2019

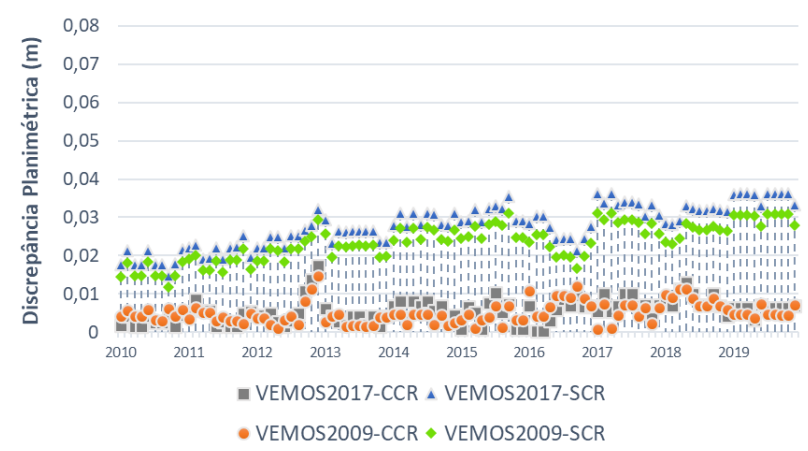

VEMOS2017 vs VEMOS2009 - Dados SALU 01/04 a 10/04 de 2010 a 2019

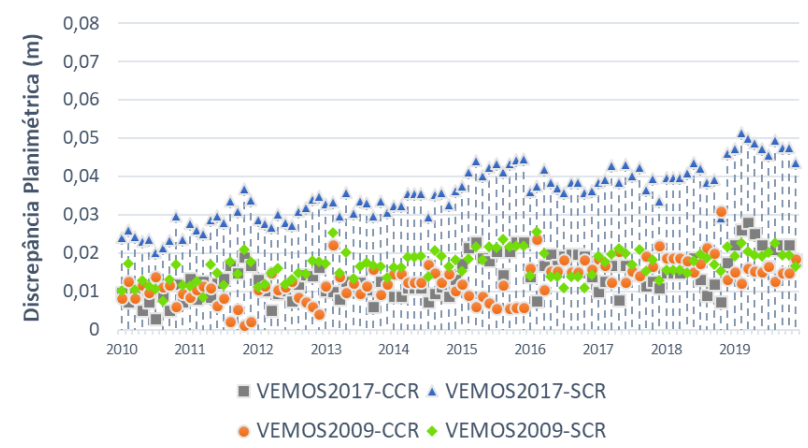

VEMOS2017 vs VEMOS2009 - Dados TOPL 01/04 a 10/04 de 2010 a 2019

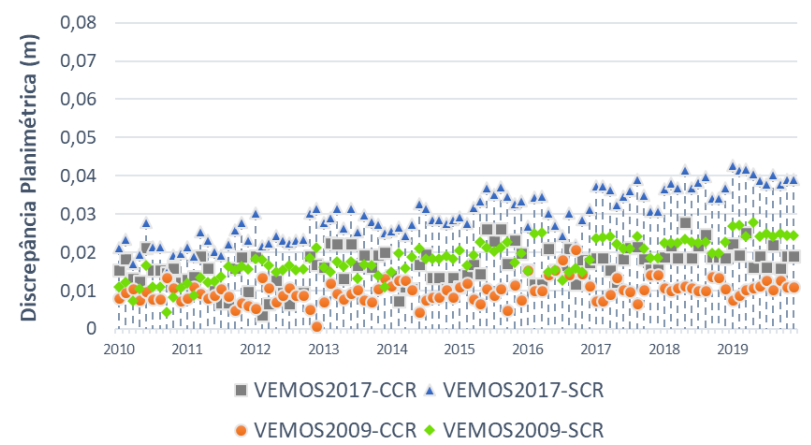

VEMOS2017 vs VEMOS2009 - Dados ROGM 01/04 a 10/04 de 2010 a 2019

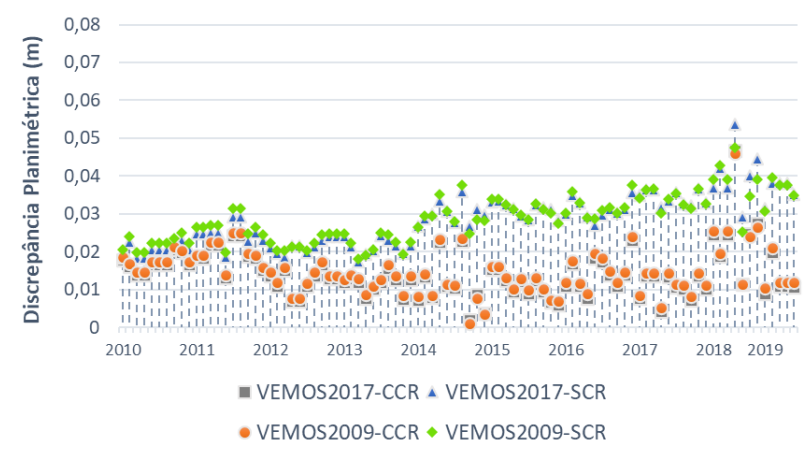

VEMOS2017 vs VEMOS2009 - Dados SMAR 01/04 a 10/04 de 2010 a 2019

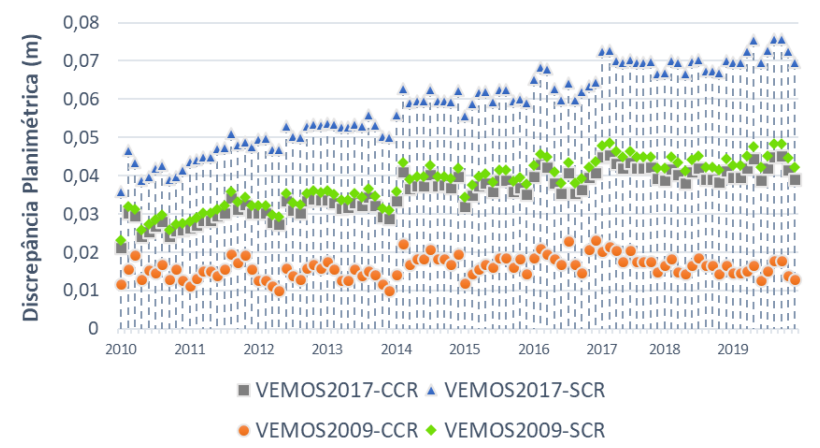

VEMOS2017 vs VEMOS2009 - Dados VICO 01/04 a 10/04 de 2010 a 2019

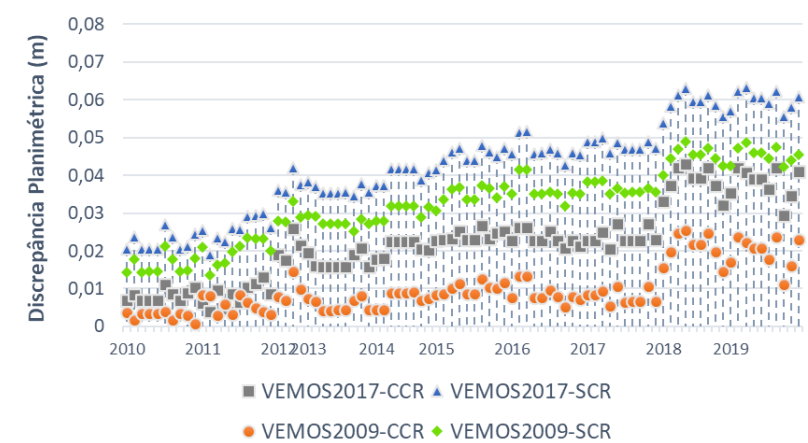

Fonte: Os autores (2021). 
Na estação SALU, assim como ocorreu na ALAR, o VEMOS2009-CCR não apresentou expressividade na redução das discrepâncias. Sua melhora máxima foi de 2,0cm $(94,74 \%)$, mínima de $0,0 \mathrm{~cm}$ e média de $0,6 \mathrm{~cm}(33,36 \%)$ quando comparado ao VEMOS2009-SCR. Particularizando para as coordenadas em que a atualização piorou a discrepância, a máxima foi de $+1,55 \mathrm{~cm}(+100,65 \%)$ e média de $+0,39 \mathrm{~cm}$ $(+30,05 \%)$ respectivamente. Por outro lado, o VEMOS2017-CCR melhorou todas as discrepâncias, com redução máxima de $3,1 \mathrm{~cm}(85,57 \%)$, mínima de $1,3 \mathrm{~cm}(44,00 \%)$ e média de $2,2 \mathrm{~cm}(62,24 \%)$ em relação ao VEMOS2017-SCR.

Analisando as discrepâncias obtidas para a estação SMAR verifica-se que o os resultados obtidos pelo VEMOS2017-CCR e o VEMOS2009-SCR foram similares e mostram uma performance mais significativa para o VEMOS2009-CCR. Salienta-se que ambos modelos apresentaram expressiva melhora após a compatibilização de referencial. O VEMOS2017-CCR obteve melhora máxima de 3,1cm (44,03\%), mínima $1,1 \mathrm{~cm}(32,03 \%)$ e média foi de 2,3cm (38,93\%) em relação ao VEMOS2017-SCR, enquanto o VEMOS2009CCR apresentou melhora máxima de 3,1cm $(69,67 \%)$, mínima de $1,1 \mathrm{~cm}(38,46 \%)$ e média de 2,2cm $(57,15 \%)$ em relação ao VEMOS2009-SCR.

Considerando a estação TOPL verifica-se que o VEMOS2009-CCR apresentou melhores resultados em $92 \%$ das coordenadas processadas, quando comparado ao VEMOS2009-SCR. Destas, a máxima melhora foi de 2,0 cm (96,23\%), mínima de $0,0 \mathrm{~cm}(0,65 \%)$ e média de $0,9 \mathrm{~cm}(46,24 \%)$. Limitando-se às discrepâncias que pioraram após a compatibilização de referencial ( $8 \%$ do total), observou-se que a média foi de $+0,37 \mathrm{~cm}$ $(+48,90 \%)$. Para o VEMOS2017, que obteve melhora em 100\% das discrepâncias, a máxima foi de $2,4 \mathrm{~cm}$ $(83,49 \%)$, mínima de $0,0 \mathrm{~cm}$ e média de $1,4 \mathrm{~cm}(44,63 \%)$.

Em relação aos resultados da VICO, verifica-se uma nítida melhora das discrepâncias após a compatibilização de referencial das velocidades, principalmente para VEMOS2009-CCR, que apresentou máxima de 3,1 cm (96,67\%), mínima de $0,6 \mathrm{~cm}(40,15 \%)$ e média de $2,2 \mathrm{~cm}(71,51 \%)$ quando comparado ao VEMOS2009-SCR. O VEMOS2017-CCR apresentou melhora máxima de 2,6cm (79,47\%), mínima de 1,2cm $(31,48 \%)$ e média de 2,0cm $(50,21 \%)$ em comparação ao VEMOS2017-SCR.

\subsection{Experimento 2}

No segundo experimento, foram realizadas quatro análises com os dados provenientes de 175 estações, com observações do dia 01/04 para todos os anos entre 2010 e 2019, adicionados aos dados já obtidos no Experimento 1, totalizando 1513 coordenadas.

Primeiramente, comparou-se as quatro velocidades entre si, ou seja, calculou-se quatro discrepâncias para cada coordenada (uma para cada velocidade), e foi registrado qual velocidade proporcionou a melhor discrepância. A junção de todos os resultados supracitados está disposta na Figura 4, que exibe a porcentagem de menores discrepâncias obtidas com cada velocidade. Nesse contexto, pode-se notar o baixo desempenho das velocidades sem compatibilização de referencial. Das coordenadas analisadas, o VEMOS2017-SCR e VEMOS2009-SCR apresentaram apenas 0,33\% e 7,21\% das menores discrepâncias. As velocidades com compatibilização obtiveram a ampla maioria das menores discrepâncias $(92,46 \%)$, sendo $62,92 \%$ referentes ao VEMOS2009-CCR e 29,54\% ao VEMOS2017-CCR.

Os dados categorizados por ano apresentaram comportamento similar ao das estações individuais, sem indicações de alguma velocidade possuir melhor desempenho em períodos específicos. Já quanto aos dados das sete estações selecionadas, pode-se notar uma grande variação do desempenho das velocidades. Nas estações SMAR e VICO, localizadas respectivamente no Rio Grande do Sul e Minas Gerais, a velocidade VEMOS2009-CCR obtiveram 100\% e 95,56\% dos melhores resultados, enquanto na estação ALAR (Alagoas) e ROGM (Rondônia) a VEMOS2017-CCR obteve 70,79\% e 92,39\% dos melhores resultados, respectivamente.

Essa variação nos resultados pode estar relacionada ao fato de os erros das velocidades do VEMOS2009 serem dependentes da densidade de observações que cobrem cada área, podendo variar de \pm 1 $\mathrm{mm} /$ ano até $\pm 9 \mathrm{~mm} / \mathrm{ano}$, de acordo com Drewes e Heidbach (2012). Os erros do VEMOS2017 também estão relacionados à densidade de cobertura, no entanto este modelo possui um maior número de estações em relação ao VEMOS2009. 
Figura 4 - Porcentagem de menores discrepâncias obtidas por cada velocidade.

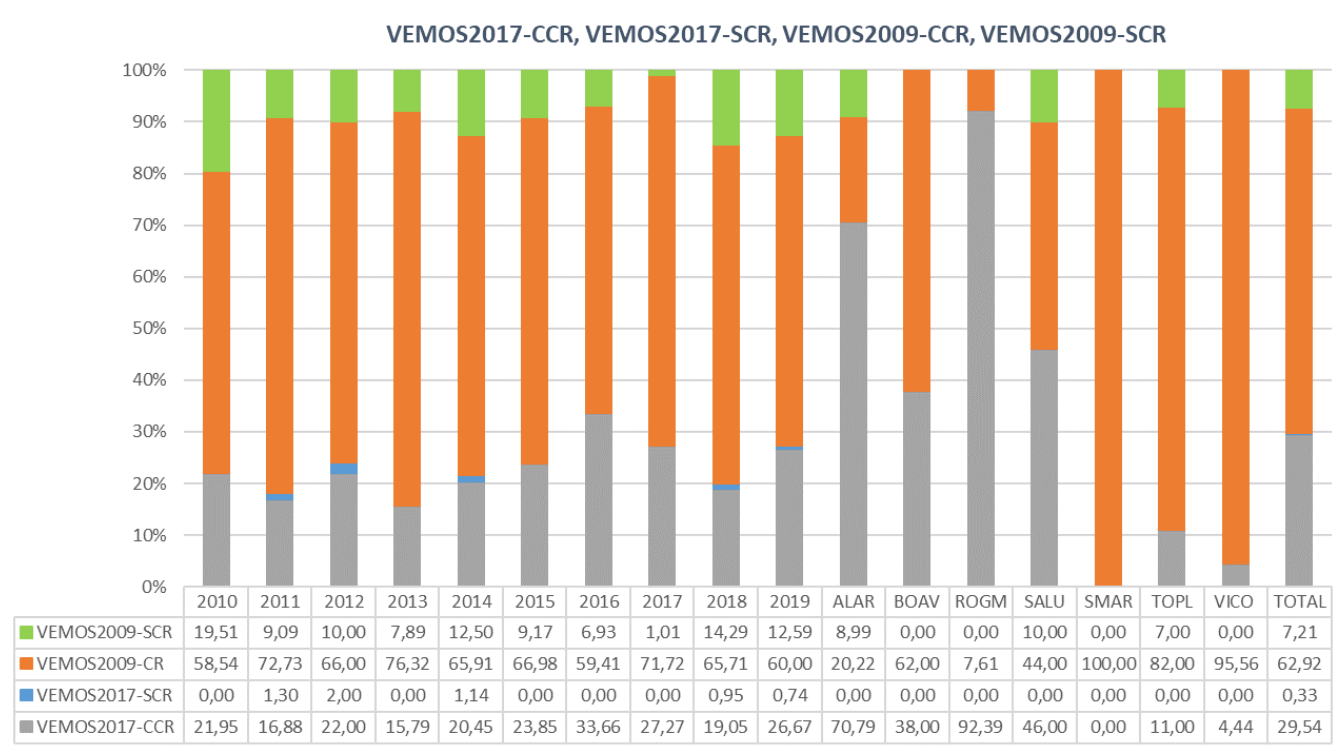

Fonte: Os autores (2021).

$\mathrm{Na}$ segunda análise confrontou-se as velocidades CCR e SCR de cada modelo VEMOS individualmente, ou seja, para cada coordenada foram obtidas duas discrepâncias com o VEMOS2017 (CCR e SCR) e duas com o VEMOS2009, e então foi registrado se a menor discrepância foi obtida com a velocidade CCR ou SCR. Desta forma, os resultados apresentados na Figura 5 comparam apenas a eficácia da compatibilização, sem comparar um modelo VEMOS ao outro.

Figura 5 - Comparação das velocidades CCR e SCR. VEMOS2009

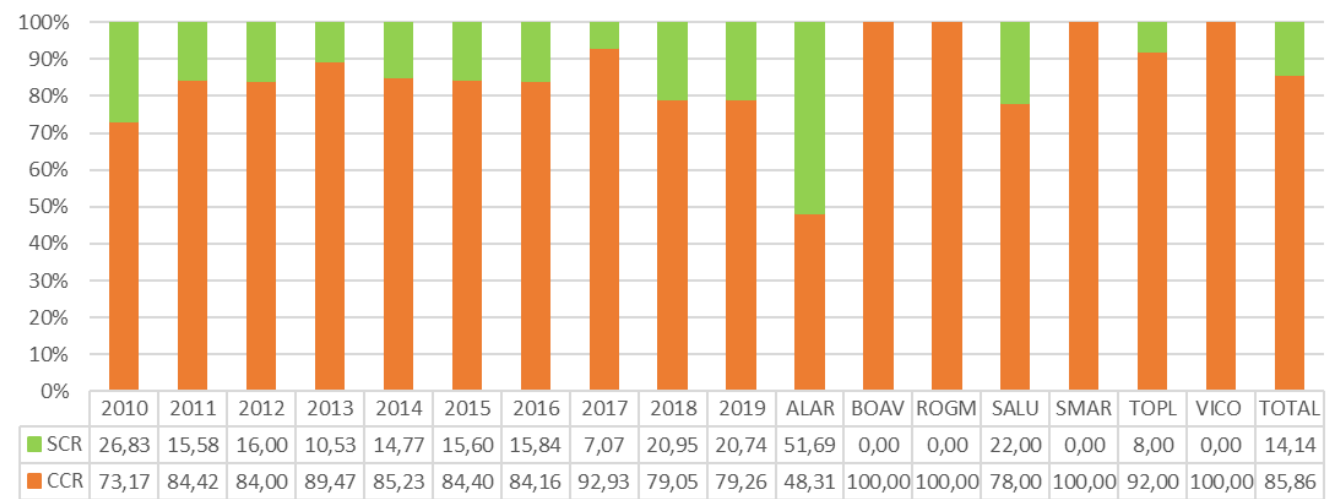

VEMOS2017

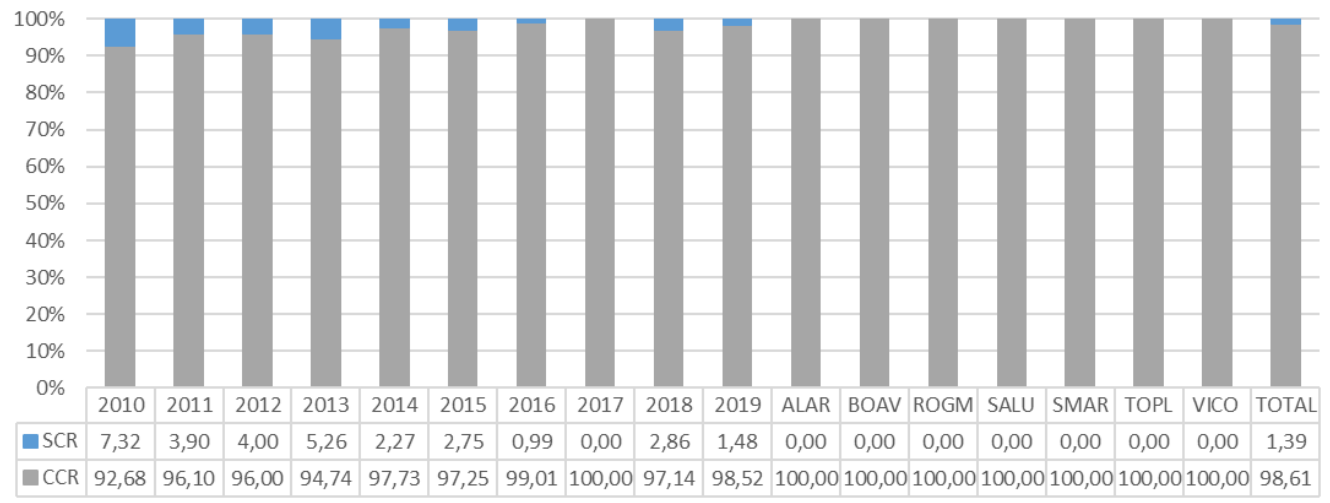

Fonte: Os autores (2021).

Analisando individualmente os modelos VEMOS2009 e VEMOS2017, fica mais uma vez evidente o impacto da compatibilização do referencial das velocidades, melhorando 98,61\% das discrepâncias utilizando 
o VEMOS2017-CCR e 85,86\% utilizando o VEMOS2009-CCR. No geral o comportamento dos dados seguiu o mesmo padrão, exceto na estação ALAR, onde 51,69\% dos melhores resultados corresponderam ao VEMOS2009-SCR.

Na terceira análise, confrontou-se os modelos VEMOS (2017 e 2009) para cada velocidade, ou seja, para cada coordenada obteve-se duas discrepâncias com velocidades CCR (uma com VEMOS2017 e uma com o VEMOS2009) e duas com velocidades SCR, registrando com qual modelo VEMOS ocorreu a menor discrepância para cada um dos casos. Desta forma, a Figura 6 exibe a porcentagem de menores discrepâncias obtidas pelo VEMOS2017 (CCR e SCR) e do VEMOS2009 (CCR e SCR).

Figura 6 - Comparação dos modelos VEMOS2017 e VEMOS2009.

CCR

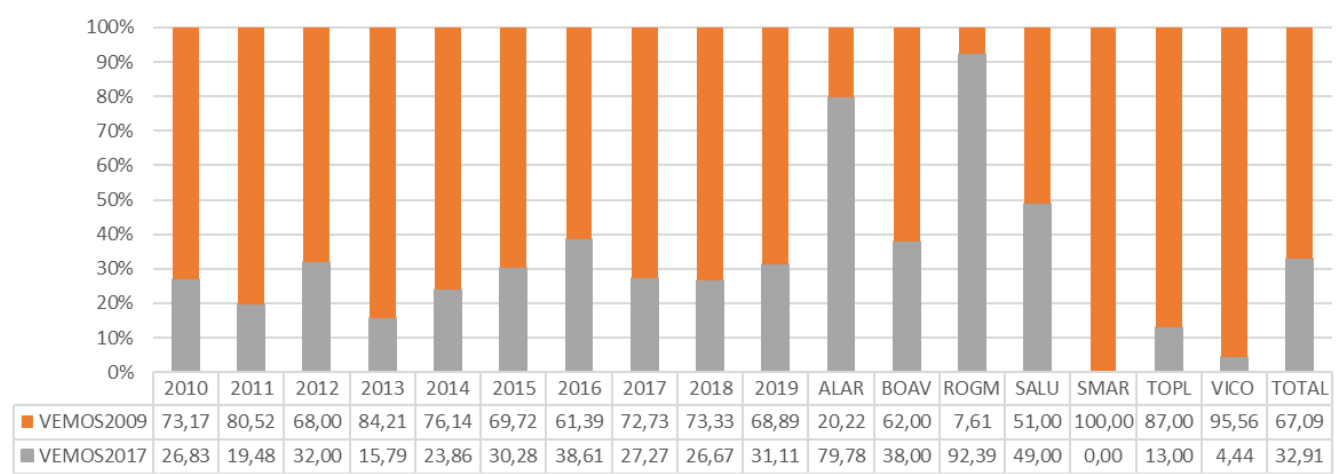

SCR

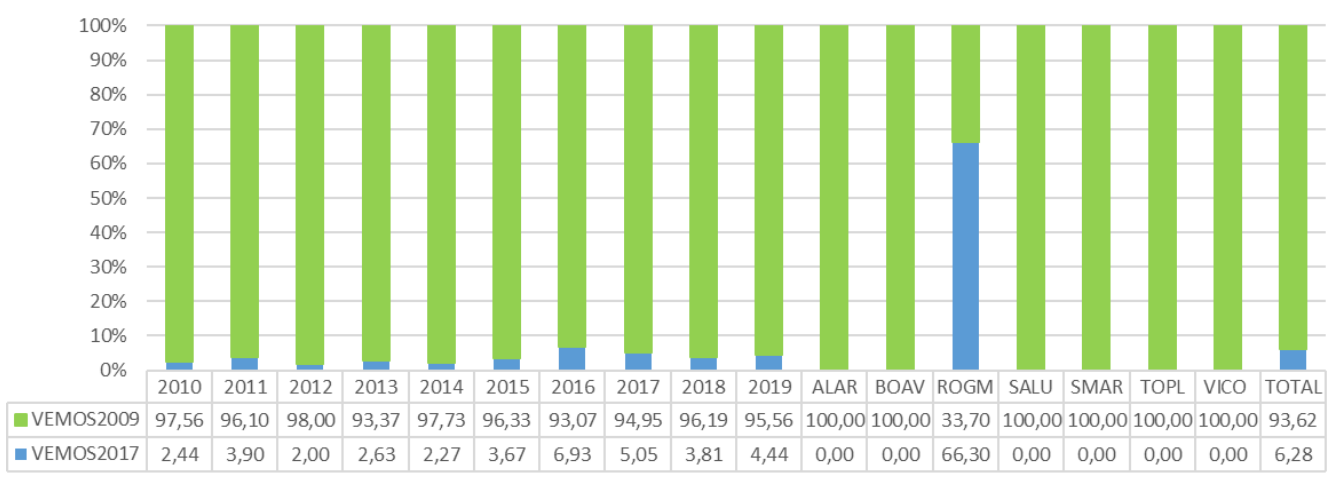

Fonte: Os autores (2021).

A partir dos resultados dispostos na Figura 6, verifica-se um melhor desempenho do VEMOS2009, que é significantemente superior para as velocidades SCR, com 93,62\% dos melhores resultados, com exceção da estação ROGM, em que o VEMOS2017-SCR apresentou resultado superior (66,30\%). Para as velocidades CCR, o VEMOS2009 apresentou 67,09\% de melhores resultados, com o VEMOS2017 sendo melhor apenas nas estações ROGM (92,39\%), e ALAR (79,78\%).

Por fim, a última análise realizada foi sobre a discrepância média dos dados utilizados nos Experimentos 1 e 2, assim como a REQM (Raiz do Erro Quadrático Médio), como mostrado na Tabela 3. 
Tabela 3 - Discrepância média e REQM dos dados analisados.

\begin{tabular}{ccccccccc}
\hline & \multicolumn{2}{c}{ VMS2017- CCR } & \multicolumn{2}{c}{ VMS2017- SCR } & \multicolumn{2}{c}{ VMS2009- CCR } & \multicolumn{2}{c}{ VMS2009-SCR } \\
& $\begin{array}{c}\text { Disc. } \text { Média } \\
(\mathbf{m})\end{array}$ & $\begin{array}{c}\text { REMQ } \\
(\mathbf{m})\end{array}$ & $\begin{array}{c}\text { Disc. Média } \\
(\mathbf{m})\end{array}$ & $\begin{array}{c}\text { REMQ } \\
(\mathbf{m})\end{array}$ & $\begin{array}{c}\text { Disc. Média } \\
(\mathbf{m})\end{array}$ & $\begin{array}{c}\text { REMQ } \\
(\mathbf{m})\end{array}$ & $\begin{array}{c}\text { Disc. Média } \\
(\mathbf{m})\end{array}$ & $\begin{array}{c}\text { REMQ } \\
(\mathbf{m})\end{array}$ \\
\hline 2010 & 0,013 & 0,002 & 0,025 & 0,002 & 0,010 & 0,001 & 0,013 & 0,001 \\
2011 & 0,015 & 0,004 & 0,028 & 0,005 & 0,010 & 0,003 & 0,016 & 0,003 \\
2012 & 0,014 & 0,002 & 0,031 & 0,003 & 0,009 & 0,001 & 0,017 & 0,001 \\
2013 & 0,017 & 0,002 & 0,034 & 0,002 & 0,009 & 0,001 & 0,019 & 0,001 \\
2014 & 0,018 & 0,005 & 0,036 & 0,006 & 0,011 & 0,003 & 0,020 & 0,003 \\
2015 & 0,018 & 0,007 & 0,038 & 0,008 & 0,013 & 0,006 & 0,022 & 0,007 \\
2016 & 0,018 & 0,005 & 0,039 & 0,007 & 0,015 & 0,008 & 0,024 & 0,008 \\
2017 & 0,020 & 0,010 & 0,045 & 0,010 & 0,013 & 0,008 & 0,029 & 0,008 \\
2018 & 0,022 & 0,008 & 0,042 & 0,007 & 0,017 & 0,004 & 0,024 & 0,008 \\
2019 & 0,022 & 0,013 & 0,045 & 0,015 & 0,018 & 0,011 & 0,027 & 0,016 \\
ALAR & 0,009 & 0,001 & 0,032 & 0,004 & 0,013 & 0,001 & 0,014 & 0,001 \\
BOAV & 0,006 & 0,001 & 0,028 & 0,003 & 0,005 & 0,001 & 0,024 & 0,002 \\
ROGM & 0,014 & 0,004 & 0,028 & 0,004 & 0,015 & 0,004 & 0,029 & 0,004 \\
SALU & 0,014 & 0,003 & 0,036 & 0,005 & 0,013 & 0,002 & 0,017 \\
SMAR & 0,036 & 0,003 & 0,059 & 0,010 & 0,016 & 0,001 & 0,038 \\
TOPL & 0,016 & 0,002 & 0,030 & 0,004 & 0,010 & 0,001 & 0,018 \\
VICO & 0,022 & 0,010 & 0,042 & 0,014 & 0,010 & 0,004 & 0,032 & 0,001 \\
\hline
\end{tabular}

Fonte: Os autores (2021).

Dessa forma, os dados de discrepância média separados tanto por anos, quanto por estações, evidenciam a diferença de desempenho das quatro velocidades, com a VEMOS2009-CCR apresentando os melhores resultados, seguida pela VEMOS2017-CCR, VEMOS2009-SCR e VEMOS2017-SCR respectivamente. As REQM das quatro velocidades mostraram comportamentos similares nos anos analisados, e assim como as discrepâncias médias apresentaram uma tendência, provavelmente devido aos movimentos da placa Sul Americana e ao maior intervalo de tempo utilizado na redução das coordenadas, o que deve ser investigado.

\subsection{Experimento 3}

Neste experimento comparou-se os resultados obtidos através da metodologia apresentada neste trabalho com a metodologia empregada pelo IBGE-PPP para a redução das coordenadas. Desta forma, calculou-se as discrepâncias das coordenadas fornecidas pelo IBG-PPP diretamente na época 2000,4 das estações ALAR, BOAV, ROGM, SALU, SMAR, TOPL e VICO referentes ao ano de 2019 e realizou-se a comparação com as discrepâncias já obtidas no Experimento 1, como mostra a Figura 7.

Figura 7 - Comparação das discrepâncias obtidas com a metodologia apresentada e com as coordenadas fornecidas pelo IBGE-PPP diretamente em 2000,4.

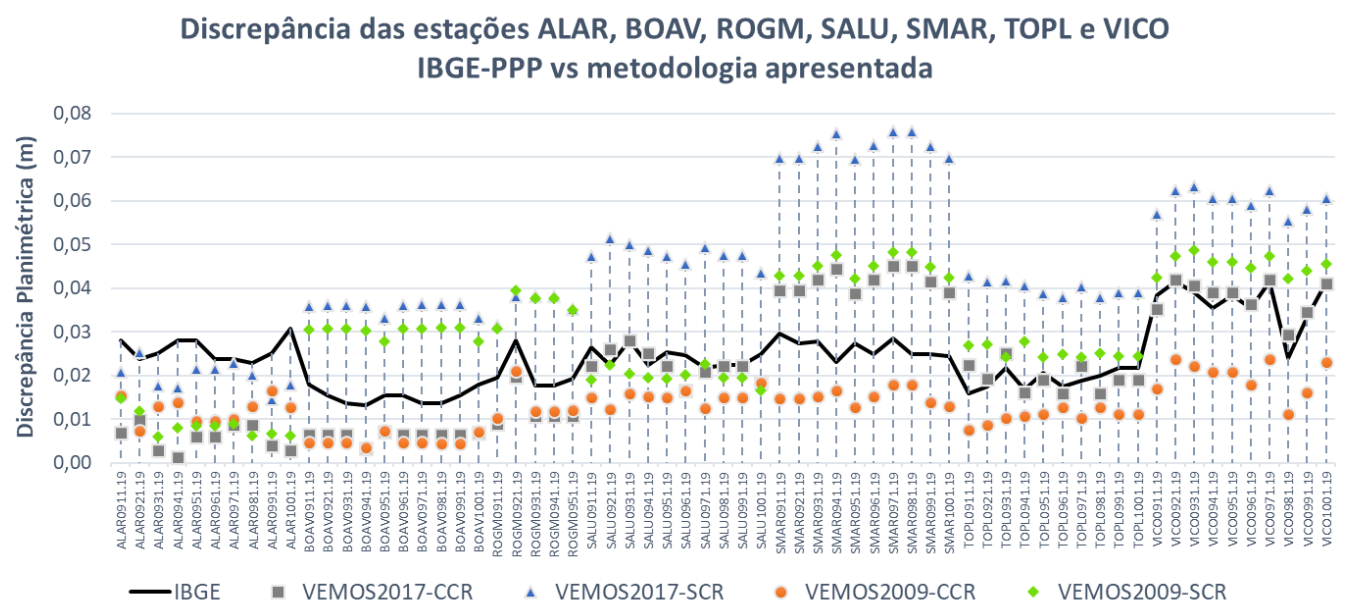

Fonte: Os autores (2021). 
As discrepâncias obtidas com a velocidade VEMOS2009-CCR apresentaram melhores resultados em relação ao IBGE-PPP em 100\% das coordenadas analisadas. Já a VEMOS2017-CCR apresentou resultados variados, com melhor performance que o IBGE-PPP nas estações ALAR, BOAV e ROGM, e pior nas estações SMAR e VICO, com as estações SALU e TOPL apresentando alternância nos resultados (ora melhor com um ora melhor com outro). Já as velocidades SCR apresentaram majoritariamente resultados piores que os do IBGE-PPP.

Analisando a média das discrepâncias e a REMQ apresentadas na Tabela 7, observa-se que o IBGEPPP performou pior que as duas velocidades CCR.

Tabela 4 - Média e REMQ das discrepâncias obtidas no experimento 3.

\begin{tabular}{cccccc}
\hline & IBGE-PPP & VEMOS2017-CCR & VEMOS2017-SCR & VEMOS2009-CCR & VEMOS2009-SCR \\
\hline Média (m) & 0,0243 & 0,0214 & 0,0452 & 0,0130 & 0,0296 \\
REMQ (m) & 0,0033 & 0,0126 & 0,0179 & 0,0016 & 0,0109 \\
\hline
\end{tabular}

Fonte: Os autores (2021).

A discrepância média da VEMOS2017-CCR foi levemente menor que a média do IBGE-PPP, mas com a REQM mais alta. A VEMOS2017-SCR apresentou a pior média e a pior REQM.

Vale destacar que apesar de o IBGE-PPP utilizar o VEMOS2009 para reduzir as coordenadas, ele não realiza o processo de compatibilização de referencial das velocidades, assim como usa parâmetros próprios para a compatibilização de referencial das coordenadas, que são diferentes aos do ITRF e não possuem variação temporal (IBGE, 2017).

\section{CONCLUSÕES}

Diante dos resultados encontrados, pôde-se verificar que a compatibilização de referencial das velocidades se mostrou eficaz para o processo de redução das coordenadas obtidas no IBGE-PPP. Das 1513 coordenadas obtidas com cada uma das quatro velocidades, a VEMOS2009-CCR apresentou 62,92\% das menores discrepâncias, seguida pela VEMOS2017-CCR com 29,54\%, VEMOS2009-SCR com 7,21\% e VEMOS2017-SCR com 0,33\%.

Considerando apenas os resultados de velocidades CCR dos dois modelos, o VEMOS2009 apresentou 67,09\% das menores discrepâncias, e o VEMOS2017 32,91\%. Para as velocidades SCR, a superioridade do VEMOS2009 foi ainda mais expressiva, possuindo 93,62\% das menores discrepâncias, contra 6,28\% do VEMOS2017.

Em relação à diminuição das discrepâncias após a compatibilização de referencial das velocidades, verificou-se que, para o VEMOS2009, 85,86\% das coordenadas apresentaram uma menor discrepância após a atualização. Já para o VEMOS2017 essa diminuição ocorreu em 98,61\% das coordenadas. Entretanto, vale destacar que a maior porcentagem de discrepâncias diminuídas pelo VEMOS2017 não necessariamente significa discrepâncias menores que as do VEMOS2009 (seja CCR ou SCR).

Salienta-se que foram analisados dados de todos os anos entre 2010 e 2019, e não foi observada nenhuma diferença significativa de performance dos modelos ao longo dos anos, mostrando que apesar do VEMOS2017 ser um modelo mais recente, não apresentou melhor desempenho em datas mais atuais. Assim como o VEMOS2009, que é um modelo baseado em observações mais antigas, não mostrou perda de desempenho em datas mais recentes.

Desta forma, recomenda-se para trabalhos que exijam maior acurácia o uso do VEMOS2009-CCR, e para trabalhos onde a melhora de resultado oferecida pelo processo de compatibilização de velocidades não seja relevante, recomenda-se o uso do VEMOS2009-SCR.

\section{Agradecimentos}

Este estudo teve o incentivo da Universidade Federal de Viçosa através do Departamento de Engenharia Civil, curso de Pós-Graduação em Engenharia Civil que disponibilizou seus equipamentos e instalações, além do apoio da Coordenação de Aperfeiçoamento de Pessoal de Nível Superior - Brasil 
(CAPES) - Código de Financiamento 001.

\section{Contribuição dos Autores}

O primeiro autor foi responsável pela curadoria dos dados, análise formal, investigação, metodologia, administração do projeto, software, validação, visualização e redação - minuta inicial. O segundo autor foi responsável pela conceptualização, metodologia, supervisão, validação e redação - revisão e edição. O terceiro autor foi responsável pelos recursos, software, visualização e redação - revisão e edição.

\section{Conflitos de Interesse}

Os autores declaram que não há conflitos de interesse.

\section{Referências}

ALTAMIMI, Z.; COLLILIEUX, X.; LEGRAND, J. GARAYT, B. \& BOUCHER, C. ITRF2005: A new release of the International Terrestrial Reference Frame based on time series of station positions and Earth Orientation Parameters, Journal of Geophysical Research, 112, B09401, DOI. 10.1029/2007JB004949. 2007.

ALTAMIMI, Z.; COLLILIEUX, X. \& MÉTIVIER, L. Analysis and results of ITRF2008. (IERS Technical Note; 37) Frankfurt am Main: Verlag des Bundesamts für Kartographie und Geodäsie, 54 pp., ISBN 978 3-86482-046-5, 2012.

ALTAMINI, Z. REBISCHUNG, P; MÉTIVIER, L; COLLILIEUX, X.. ITRF2014: A new release of the International Terrestrial. Journal of Geophysical Research: Solid Earth. 2016.

ALMEIDA, M. S.; OLIVEIRA, G. D.; DAL POZ, W. R. Comparação de coordenadas de estações da RBMC transformadas e atualizadas para o ITRF2014. In: SIMPÓSIO BRASILEIRO DE CIÊNCIAS GEODÉSICAS E TECNOLOGIAS DA GEOINFORMAÇÃO, 6., 2016, Recife. Anais... Presidente Prudente - SP, 2017. p. 543-550.

BRAGA, F. L. S.; DAL POZ, W. R. Mudança de referencial e atualização de coordenadas entre o IGS14 (ITRF2014) e o SIRGAS2000 (ITRF2000). In: SIMPÓSIO BRASILEIRO DE GEOMÁTICA, 4., Presidente Prudente, 2017. Anais... Presidente Prudente: UNESP, 2017. p .543-550.

BRAGA, F. L. S.; DAL POZ, W. R. Potencialidades do PPP no Software Bernese com Compatibilizações de Sistemas Geodésicos de Referência e de Tempo. Revista Brasileira de Cartografia, v. 71, n. 3, p. 726755, 25 set. 2019.

BRUNINI, C.; SÁNCHEZ, L. Geodetic Reference Frame for the Americas: advancing Geodesy in Latin America and the Caribbean. GIM Internacional. Estados Unidos, v. 27, p. 26-31, 2015.

CARVALHO, A. S. Implicações do emprego de diferentes modelos de velocidades e parâmetros da transformação de Helmert no posicionamento geodésico na placa sul-americana. $188 \mathrm{f}$. Tese (Doutorado em Engenharia Civil) - Universidade Federal de Viçosa, Viçosa. 2015.

CARVAlHO, A. S.; DAL POZ, W. R.; LAROCCA, A. P. C. Compatibilização de Referenciais de Coordenadas e Velocidades Com Estimativa de Precisão. Boletim de Ciências Geodésicas, [S.1.], v. 21, n. 3, sep. 2015. ISSN 1982-2170. Disponível em: 〈https://revistas.ufpr.br/bcg/article/view/43215>. Acesso em: 26 jul. 2020.

DREWES H., HEIDBACH O. (2005). Deformation of the South American crust estimated from finite element and collocation methods. In: Sansò F. (Ed.) A Window on the Future of Geodesy, IAG Symposia 128: 544-549, Springer, doi: 10.1007/3-540-27432-4_92.

DREWES H., HEIDBACH O. (2012). The 2009 Horizontal Velocity Field for South America and the Caribbean. In: Kenyon S., M.C. Pacino, U. Marti (Eds.), "Geodesy for Planet Earth", IAG Symposia, 136: 657-664, DOI. 10.1007/978-3-642-20338-1_81. 
DREWES H. AND SÁNCHEZ L. (2020): Velocity model for SIRGAS 2017: VEMOS2017, doi: 10.1594/pangaea.912350, Technische Universitaet Muenchen, Deutsches Geodaetisches Forschungsinstitut (DGFI-TUM), IGS RNAAC SIRGAS, supplement to: SÁNCHEZ L., DREWES H. (2020): Geodetic monitoring of the variable surface deformation in Latin America. International Association of Geodesy Symposia Series, Vol 152, doi: 10.1007/1345_2020_91.

INSTITUTO BRASILEIRO DE GEOGRAFIA E ESTATÍSTICA (IBGE). Nota Técnica. Término do período de transição para adoção no Brasil do Sistema de Referência Geocêntrico para as Américas (SIRGAS), em sua realização de 2000,4 (SIRGAS2000). 2015. Disponível em: <http://geoftp.ibge.gov.br/metodos_e_outros_documentos_de_referencia/normas/nota_tecnica_termino_ periodo_transicao_sirgas2000.pdf>. Acesso em: 03 mai. 2020.

INSTITUTO BRASILEIRO DE GEOGRAFIA E ESTATÍSTICA (IBGE). Manual do usuário aplicativo online IBGE-PPP. Rio de Janeiro: 2017. Brasil, 2017. Disponível em: <https://biblioteca.ibge.gov.br/visualizacao/livros/liv101677.pdf>. Acesso em: 21 mai. 2020.

INSTITUTO BRASILEIRO DE GEOGRAFIA E ESTATÍSTICA-PPP (IBGE-PPP). Serviço online para pós-processamento de dados GNSS - IBGE-PPP. 2020. Disponível em: < https://www.ibge.gov.br/geociencias/informacoes-sobre-posicionamento-geodesico/servicos-paraposicionamento-geodesico/16334-servico-online-para-pos-processamento-de-dados-gnss-ibgeppp.html?=\&t=saiba-mais-geociencias>. Acesso em: 03 jun. 2020.

INTERNATIONAL GNSS SERVICE ANALYSIS CENTER COORDINATOR (IGS ACC). Chronology of IGS Reference Frame Usage. 2020. Disponível em: < http://acc.igs.org/igs-frames.html>. Acesso em 21 mai. 2020.

JEKELI, C. Geometric reference systems in geodesy. Division of Geodesy and Geospatial Science, School of Earth Sciences, OST - Ohio State University, 209 p., 2012.

MOREIRA, D. M. Rede de Referência Altimétrica para Avaliação da Altimetria por Satélites e Estudos Hidrológicos na Região Amazônica. 175 p. Dissertação (Mestrado em Engenharia Civil) - Universidade Federal do Rio de Janeiro. Rio de Janeiro, RJ, 2010.

RAMOS, DAL POZ e CARVALHO, 2016. Análise Das Possibilidades De Transformação De Referencial E Atualização De Coordenadas Estimadas Pelo PPP. Boletim de Ciências Geodésicas. Curitiba, v. 22, n. 3 , 2016. Disponível em: <https://revistas.ufpr.br/bcg/article/view/48684/29281>. Acesso em: 01 nov. 2019.

REBISCHUNG, P.; SCHMID, R.. IGS14/igs14.atx: a new Framework for the IGS Products. 2016. American Geophysical Union. Disponível em: 〈https://mediatum.ub.tum.de/doc/1341338/1341338.pdf〉. Acesso em 27 jul. 2020.

REBISCHUNG, P. e SCHMID, R. Switch to IGb14 reference frame. 2020. Disponível em: < https://lists.igs.org/pipermail/igsmail/2020/007917.html?fbclid=IwAR2BtFMNHlgczy31QYqv4rLCa6N ZXj_hJvg66ubDdD0VJ1vEOxrHMEfNeTs>. Acesso em: 3 jun. 2020.

SÁNCHEZ L., DREWES H. (2016): VEMOS2015: Velocity and deformation model for Latin America and the Caribbean, doi: 10.1594/pangaea.863131, supplement to: SÁNCHEZ L. DREWES H. (2016): Crustal deformation and surface kinematics after the 2010 earthquakes in Latin America. Journal of Geodynamics, doi: 10.1016/j.jog.2016.06.005.

SAPUCCI, L. F.; MONICO, J. F. G. Transformação de Helmert generalizada no posicionamento de alta precisão: fundamentação teórica e exemplificações. Revista Brasileira de Geofísica. Presidente PrudenteSP, v.18, n.2, pp.161-172, 2001. DOI: https://doi.org/10.1590/S0102-261X2000000200005.

SILVA, A. L.; COSTA, S. M. A.; VAZ, J. A. Deslocamento das estações SIRGAS-CO em função do terremoto ocorrido no chile - uma abordagem do centro de processamento SIRGAS - IBGE. In: III Simpósio Brasileiro de Ciências Geodésicas e Tecnologias da Geoinformação. Anais... Recife - PE, 2730 de julho de 2010. p. 001 de 003.

SISTEMA DE REFERÊNCIA GEOCÊNTRICO PARA AS AMÉRICAS (SIRGAS). Modelo de velocidades para o SIRGAS. 2020. Disponível em: <http://www.sirgas.org/pt/sirgas-con-network/velocity-model/>. Acesso em: 27 fev. 2020. 
SOLER, T; SNAY, R. A. Transforming positions and velocities between the International Terrestrial Reference Frame of 2000 e North American datum of 1983. Journal of Surveying Engineering. Estados Unidos, v. 130, n. 2, p. 49-55, 2004.

PETTIT. G. E LUZUM. B. IERS Nota Técnica 36. Frankfurt am Main: Verlag des Bundesamts für Kartographie und Geodäsie, 2010. 179 pp. Disponível em: <https://www.iers.org/IERS/EN/Publications/TechnicalNotes/tn36.html>. Acesso em: 23 abr. 2020.

PROL, F. S.; JUNIOR, J. M.; NIEVINSKI, F. G.; GOMES, R. L. E FILHO, A. C. P..Transformação Entre Referenciais e Cálculo de Velocidades Através do Aplicativo Web Trevel. Revista Brasileira de Cartografia. Uberlândia, n. 66/3, p. 569-579, 2014.

TORGE, W. Geodesy. $3^{\text {a }}$ edição. Berlim-New York: Walter de Gruyter, 2001.

WESTON, N. D. e SOLER, T. Rigorous Geodetic Positioning in the Americas. In: FIG REGIONAL CONFERENCE, 8, 2012. Montevideo, Uruguay, Anais... Montevideo. 2012. p. 1-13.

\section{Biografia do autor principal}

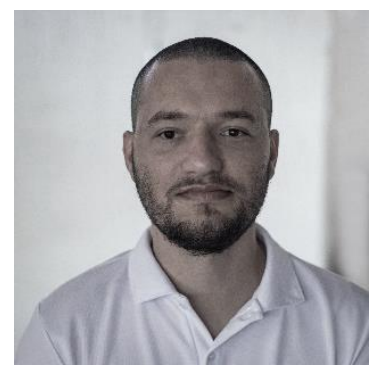

Krisley Xavier Soares de Freitas é natural de Viçosa-MG. Formado em Engenharia de Agrimensura e Cartográfica pela Universidade Federal de Viçosa (2018), pósgraduado em Geoprocessamento e Georreferenciamento pela Faculdade Única de Ipatinga (2018), atualmente é mestrando em Engenharia Civil, área de Concentração em Informações Espaciais (Geodésia) pela Universidade Federal de Viçosa.

Esta obra está licenciada com uma Licença Creative Commons Atribuição 4.0 Internacional - CC BY. Esta licença permite que outros distribuam, remixem, adaptem e criem a partir do seu trabalho, mesmo para fins comerciais, desde que lhe atribuam o devido crédito pela criação original. 